\title{
Rickettsia asiatica sp. nov., isolated in Japan
}

\author{
Correspondence \\ Pierre-Edouard Fournier \\ pierre-edouard.fournier@ \\ medecine.univ-mrs.fr
}

\author{
Hiromi Fujita, ${ }^{1} \dagger$ Pierre-Edouard Fournier, ${ }^{2} \dagger$ Nobuhiro Takada, ${ }^{3}$ \\ Takehiro Saito ${ }^{4}$ and Didier Raoult ${ }^{2}$ \\ 1'Ohara Research Laboratory, Ohara General Hospital, Fukushima 960-0195, Japan \\ ${ }^{2}$ Unité des rickettsies, IFR 48, CNRS UMR 6020, Faculté de médecine, Université de la \\ Méditerranée, 27 Boulevard Jean Moulin, 13385 Marseille cedex 05, France \\ ${ }^{3}$ Department of Pathological Sciences, Faculty of Medical Sciences, University of Fukui, \\ Matsuoka, Fukui 910-1193, Japan \\ ${ }^{4}$ Aizawa Hospital, 2-5-1 Honjo, Matsumoto 390-8510, Japan
}

In Japan, the discovery of Rickettsia japonica (Uchida et al., 1992) as the agent of Japanese spotted fever (Mahara et al., 1985) has motivated the study of spotted fever group (SFG) rickettsiae in ticks. To date, 63 SFG rickettsial strains have been isolated from ticks in this country (Fujita et al., 1999). Among these, only three Rickettsia species with validly published names have been identified, namely, $R$. japonica, Rickettsia helvetica and Rickettsia tamurae. R. japonica has been identified in several tick species including Haemaphysalis longicornis, Haemaphysalis flava, Haemaphysalis formosensis, Haemaphysalis hystricis, Dermacentor taïwanensis and Ixodes ovatus (Mahara, 1997; Fournier et al., 2002). R. helvetica has been found in Ixodes monospinosus and Ixodes persulcatus (Fournier et al., 2002) and R. tamurae has been isolated from Amblyomma testudinarium (Fournier et al., 2006). In June 1993, a rickettsial isolate named IO- ${ }^{\mathrm{T}}$ was obtained from an $I$. ovatus nymph collected in Oozaso, in the Fukushima prefecture (Fujita et al., 1999). Subsequently, 32 additional isolates that exhibited gltA gene sequences identical to that of the IO- $1^{\mathrm{T}}$ isolate have been obtained from I. ovatus ticks in Japan. To date, this rickettsia has not been found in any other

†These authors contributed equally to this work.

Abbreviations: MIF, microimmunofluorescence; SFG, spotted fever group.

The GenBank/EMBL/DDBJ accession numbers for the $16 \mathrm{~S}$ rRNA, gltA, ompB and sca4 gene sequences of strain $10-1^{\top}$ are AF394906, AF394901, DQ1 10870 and DQ110869, respectively. tick species. A preliminary mouse serotyping assay and sequencing of the entire 16S rRNA (rrs) and gltA genes demonstrated that Rickettsia sp. strain IO- $1^{\mathrm{T}}$ exhibits a unique serotype and unique genotypic characteristics (Takada et al., 2001; Fournier et al., 2002). An electron microscopic study of Rickettsia sp. strain IO- $1^{\mathrm{T}}$ demonstrated that it multiplied in the cytoplasm of L929 cells, although a few bacteria were seen in intranuclear location (Yano et al., 2004). However, the ability of this rickettsia to stimulate actin-based motility, as described for other SFG rickettsiae (Heinzen et al., 1993), has not been investigated to date. We herein demonstrate that this rickettsia fulfils the requirements for its proposal as a novel species (Fournier et al., 2003; Raoult et al., 2005).

Five IO-1-like rickettsial isolates obtained from Japanese $I$. ovatus ticks were included in our study. These included strain $\mathrm{IO}-1^{\mathrm{T}}$ described above, strain IO-2 from the Kagoshima prefecture (isolated in 1994), strain IO-12 from the Fukushima prefecture (1997), strain IO-25 from the Akita prefecture (1997) and strain IO-38 from Awaji island (2001). For strain IO- $1^{\mathrm{T}}$, we first retrieved $r r s$ and $g l t A$ nucleotide sequences from GenBank (Table 1). We then extracted DNA from strain IO- $1^{\mathrm{T}}$ and the other four isolates using the QIAmp tissue kit (QIAGEN), according to the manufacturer's instructions. PCR amplification and sequencing of the $5^{\prime}$ and $3^{\prime}$-ends of the ompA gene and the complete $o m p B$ and $s c a 4$ genes were attempted from all five isolates using previously described primers and 
Table 1. Accession numbers of the gene sequences used in the present study

NA, Not available.

\begin{tabular}{|c|c|c|c|c|}
\hline Strain & $16 S$ rRNA & gltA & $o m p B$ & sca4 \\
\hline R. asiatica sp. nov. $\mathrm{IO}-1^{\mathrm{T}}$ & AF394906 & AF394901 & DQ110870 & DQ110869 \\
\hline R. aeschlimannii $\mathrm{MC}^{\mathrm{T}}{ }^{\mathrm{T}}$ & U74757 & U59722 & AF123705 & AF163005 \\
\hline R. africae ESF-5 & L36098 & U59733 & AF123706 & AF151724 \\
\hline R. akari MK $(\text { Kaplan })^{\mathrm{T}}\left(=\right.$ ATCC VR- $\left.148^{\mathrm{T}}\right)$ & L36099 & U59717 & AF123707 & AF213016 \\
\hline R. australis NIAID Phillips $32^{\mathrm{T}}$ & L36101 & U59718 & AF123709 & AF187982 \\
\hline R. bellii 369L42-1 & L36103 & U59716 & NA & NA \\
\hline R. canadensis $2678^{\mathrm{T}}$ & L36104 & U59713 & NA & $\mathrm{NA}$ \\
\hline R. conorii NIAID Malish $7^{\mathrm{T}}\left(=\mathrm{ATCC}\right.$ VR- $\left.613^{\mathrm{T}}\right)$ & AF541999 & U59730 & AF123721 & AF163008 \\
\hline R. felis URRWXCal2 (=ATCC VR-1525) & L28944 & AF210692 & AF210695 & AF196973 \\
\hline R. helvetica С9Р9 & L36212 & U59723 & AF123725 & AF163009 \\
\hline R. honei TT-118 (=ATCC VR-599) & L36220 & U59726 & AF123724 & AF163004 \\
\hline R. japonica YM & L36213 & U59724 & AF123713 & AF155055 \\
\hline R. massiliae Mtu1 ${ }^{\mathrm{T}}$ & L36214 & U59719 & AF123714 & AF163003 \\
\hline R. montanensis $\mathrm{M} / 5-6$ & L36215 & U74756 & AF123716 & AF163002 \\
\hline R. parkeri NIAID maculatum $20^{\mathrm{T}}$ & L36673 & U59732 & AF123717 & AF155059 \\
\hline R. peacockii Skalkaho ${ }^{\mathrm{T}}$ & U55820 & AY590152 & NA & NA \\
\hline R. prowazekii Brein $1^{\mathrm{T}}\left(=\right.$ ATCC VR- $\left.142^{\mathrm{T}}\right)$ & M21789 & M17149 & AF123718 & AF200340 \\
\hline R. rhipicephali Burgdorfer 3-7-female $6^{\mathrm{T}}$ & L36216 & U59721 & AF123719 & AF155053 \\
\hline R. rickettsii $\mathrm{R}$ (Bitteroot) (=ATCC VR-891) & L36217 & U59729 & X16353 & AF163000 \\
\hline R. sibirica $246^{\mathrm{T}}\left(=\right.$ ATCC VR- $\left.151^{\mathrm{T}}\right)$ & L36218 & U59734 & AF123722 & AF155057 \\
\hline R. slovaca $13-\mathrm{B}$ & L36224 & U59725 & AF123723 & AF155054 \\
\hline R. tamurae $\mathrm{AT}-1^{\mathrm{T}}$ & AY049981 & AF394896 & DQ113910 & DQ113911 \\
\hline R. typhi Wilmington ${ }^{\mathrm{T}}\left(=\right.$ ATCC VR-144 $\left.{ }^{\mathrm{T}}\right)$ & L36221 & U59714 & L04661 & AF188482 \\
\hline
\end{tabular}

conditions (Fournier et al., 1998; Roux \& Raoult, 2000; Sekeyova et al., 2001). In addition, rrs and gltA genes were amplified from strains IO-2, IO-12, IO-25 and IO-38. No ompA PCR product could be obtained from any of the studied isolates. However, whether this negative result is the result of the absence of the ompA gene in IO-1-like isolates or the incapacity of the primers used to amplify the gene is not yet known. In contrast, PCR products of the expected sizes were obtained for the other genes. Sequences were edited by removal of regions of ambiguity at the $5^{\prime}$ and $3^{\prime}$ ends so that their lengths were 1417, 1047, 4845 and 2946 bp for $r r s, g l t A, o m p B$ and $s c a 4$, respectively. The five isolates exhibited identical sequences for each of the four genes. For further tests, we selected the IO- $1^{\mathrm{T}}$ strain as representative of these five isolates. The phylogenetic relationships of Rickettsia sp. strain IO- $1^{\mathrm{T}}$ with all Rickettsia species with validly published names for which $r r s, g l t A, o m p B$ and $s c a 4$ gene sequences are available (Table 1) were evaluated for each gene using the neighbour-joining and maximumparsimony methods within the MEGA 3.1 software (Kumar et al., 2004) and the maximum-likelihood method within the PHYLIP software package (Felsenstein, 1989). For all studied genes and using the three analysis methods, Rickettsia sp. strain $\mathrm{IO}-1^{\mathrm{T}}$ was grouped with $R$. helvetica with elevated bootstrap values (Fig. 1). When calculating similarity values between nucleotide sequences of strain IO$1^{\mathrm{T}}$ and Rickettsia species, transitions or transversions, not insertions or deletions, were included. For all four loci examined, strain IO- $1^{\mathrm{T}}$ shared highest sequence similarity with $R$. helvetica $(99 \cdot 0,99 \cdot 0,98 \cdot 2$ and $98 \cdot 3 \%$, respectively, for the rrs, gltA, ompB and sca4 genes). However, these values were lower than the cut-offs proposed for Rickettsia species definition (Fournier et al., 2003). Therefore, on the basis of genotypic criteria, Rickettsia sp. strain IO- $1^{\mathrm{T}}$, although phylogenetically closely related to the R. helvetica species (Fig. 1), belonged to a distinct species. In addition, we compared Rickettsia sp. strain IO- $1^{\mathrm{T}}$ to another Japanese rickettsia, $R$. tamurae, also phylogenetically related to $R$. helvetica (Fournier et al., 2006). We found degrees of nucleotide sequence similarity between the two rickettsiae of $98 \cdot 5,96 \cdot 7,95 \cdot 7$ and $91 \cdot 2 \%$, respectively, for the $r r s, g l t A$, ompB and sca 4 genes. Therefore, the two rickettsiae were sufficiently genetically different to be classified within different species. Mouse serotyping was conducted by microimmunofluorescence (MIF) as described by Philip et al. (1978). We used as antigens Rickettsia sp. strain IO- ${ }^{\mathrm{T}}$, $R$. helvetica type strain $\mathrm{C} 9 \mathrm{P} 9^{\mathrm{T}}$ and $R$. tamurae type strain AT$1^{\mathrm{T}}$, cultivated on Vero cells (ATCC CRL-1587) as described previously (Marrero \& Raoult, 1989). Strain IO- $1^{\mathrm{T}}$ caused cytopathic effects after 5 days of incubation. If the specificity difference (SPD) was $\geqslant 3$, the isolates were assumed to belong to different serotypes. Using serum from mice immunized with strain IO- ${ }^{\mathrm{T}}$, we found MIF antibody titres of $1: 400,1: 50$ and $1: 100$ to the homologous antigen and to $R$. tamurae and $R$. helvetica, respectively. Using serum from mice immunized with $R$. tamurae, we found MIF antibody 


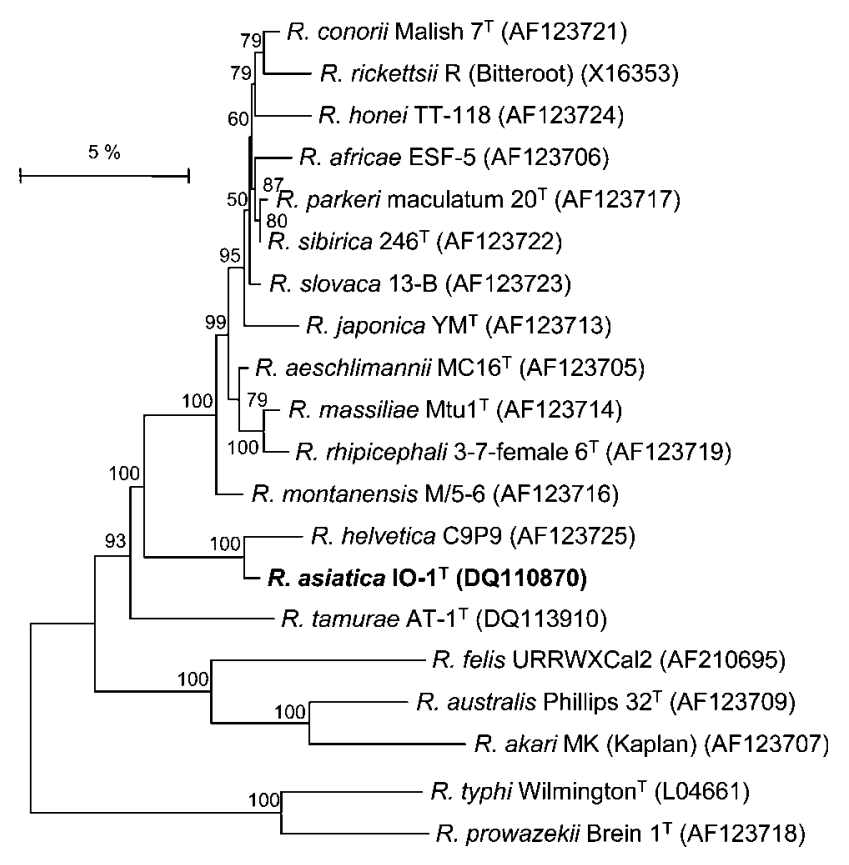

Fig. 1. Unrooted dendrogram showing the phylogenetic position of Rickettsia sp. strain $1 \mathrm{O}-1^{\top}$ among Rickettsia species with validly published names inferred from the comparison of $o m p B$ gene sequences by the neighbour-joining method. GenBank accession numbers are indicated in parentheses. Bootstrap values are indicated at nodes. Bar, 5\% nucleotide sequence divergence.

titres of $1: 400,1: 50$ and $1: 100$ to the homologous antigen and to strain $\mathrm{IO}-1^{\mathrm{T}}$ and $R$. helvetica, respectively. Finally, using serum from mice immunized with $R$. helvetica, we found MIF antibody titres of $1: 800,1: 200$ and $1: 100$ to the homologous antigen and to strain $\mathrm{IO}-1^{\mathrm{T}}$ and $R$. tamurae antigens, respectively. On the basis of these results, the SPD between these rickettsiae was 4 between strain $\mathrm{IO}-1^{\mathrm{T}}$ and $R$. helvetica and 6 between strain $\mathrm{IO}-1^{\mathrm{T}}$ and $R$. tamurae. Therefore, the genotypic and serotypic specificity of Rickettsia sp. strain $\mathrm{IO}-1^{\mathrm{T}}$ justify its classification in a distinct species.

Our data support the proposal to classify Rickettsia sp. strain IO- $1^{\mathrm{T}}$ within a new species. Thus, we formally propose the creation of Rickettsia asiatica sp. nov., which contains the strains $\mathrm{IO}-1^{\mathrm{T}}$, IO-2, IO-12, IO-25 and IO-38. This rickettsia has only been found in Japan to date. Following discussions held at the meeting of the International Committee on Systematics of Prokaryotes (ICSP) and its Judicial Commission (JC) in San Francisco, 2005, and in anticipation of the published minutes of the JC and ICSP, a committee consisting of the chairman of the ICSP, the chairman of the Judicial Commission of the ICSP and the Editor of the International Journal of Systematic and Evolutionary Microbiology has granted an exception in this case to the Rule governing the deposit of type material in two different collections in two different countries.

\section{Description of Rickettsia asiatica sp. nov.}

Rickettsia asiatica (a.si.a'ti.ca. L. fem. adj. asiatica Asian).

Gram-negative, obligately intracellular bacterium. Grows in Vero and L929 cells at $32{ }^{\circ} \mathrm{C}$ in minimal essential medium supplemented with $2 \%$ fetal calf serum and $2 \mathrm{mg} \mathrm{L-}$ glutamine $\mathrm{ml}^{-1}$. Non motile. 16S rRNA, gltA, ompB and sca4 gene sequencing indicate that this rickettsia is clearly different from all other recognized rickettsial species, the most closely related organisms being $R$. helvetica and $R$. tamurae. No information is available about the possible pathogenicity of this organism for vertebrate hosts. The known geographical distribution of this bacterium is restricted to Japan.

The type strain is strain IO- $1^{\mathrm{T}}\left(=\mathrm{CSUR} \mathrm{R} 2^{\mathrm{T}}\right.$ ), which was isolated from Ixodes ovatus ticks in 1993 in Fukushima prefecture, Japan (Fujita et al., 1999). The type strain has been deposited in the Collection de souches de l'Unite des rickettsies (CSUR), WHO Collaborative Center for Rickettsioses, Borrelioses and Tick-borne Infections, Marseilles, France, and is being deposited in the American Type Culture Collection (ATCC). Strains IO2, IO-12, IO-25 and IO-38, have been deposited in the CSUR under references CSUR R4, CSUR R5, CSUR R6 and CSUR R7, respectively, and are also being deposited in the ATCC.

\section{Acknowledgements}

The work carried out in Japan was supported in part by Grants-in-Aid for Scientific Research from the Japan Society for the Promotion of Science (no. 13576005 and 16406008).

\section{References}

Felsenstein, J. (1989). PHYLIP - phylogeny inference package (version 3.2). Cladistics 5, 164-166.

Fournier, P.-E., Roux, V. \& Raoult, D. (1998). Phylogenetic analysis of spotted fever group rickettsiae by study of the outer surface protein rOmpA. Int J Syst Bacteriol 48, 839-849.

Fournier, P.-E., Fujita, H., Takada, N. \& Raoult, D. (2002). Genetic identification of rickettsiae isolated from ticks in Japan. J Clin Microbiol 40, 2176-2181.

Fournier, P.-E., Dumler, J. S., Greub, G., Zhang, J., Wu, Y. \& Raoult, D. (2003). Gene sequence-based criteria for identification of new Rickettsia isolates and description of Rickettsia heilongjiangensis sp. nov. J Clin Microbiol 41, 5456-5465.

Fournier, P.-E., Takada, N., Fujita, H. \& Raoult, D. (2006). Rickettsia tamurae sp. nov., isolated from Amblyomma testudinarium ticks. Int J Syst Evol Microbiol 56, 1673-1675.

Fujita, H., Watanabe, Y., Ishikura, M. \& Takada, N. (1999). List of all isolates of spotted fever group Rickettsiae from ticks in Japan 1993-1998. Ann Rep Ohara Hosp 42, 45-50.

Heinzen, R. A., Hayes, S. F., Peacock, M. G. \& Hackstad, T. (1993). Directional actin polymerization associated with spotted fever group rickettsia infection of Vero cells. Infect Immun 61, 1926-1935. 
Kumar, S., Tamura, K. \& Nei, M. (2004). MEGA3: integrated software for molecular evolutionary genetics analysis and sequence alignment. Brief Bioinform 5, 150-163.

Mahara, F. (1997). Japanese spotted fever: report of 31 cases and review of the literature. Emerg Infect Dis 3, 105-111.

Mahara, F., Koga, K., Sawada, S. \& 8 other authors (1985). The first report of the rickettsial infections of spotted fever group in Japan; three clinical cases. Jpn J Assoc Infect Dis 59, 1165-1172.

Marrero, M. \& Raoult, D. (1989). Centrifugation-shell vial technique for rapid detection of Mediterranean spotted fever rickettsia in blood culture. Am J Trop Med Hyg 40, 197-199.

Philip, R. N., Casper, E. A., Burgdorfer, W., Gerloff, R. K., Hugues, L. E. \& Bell, E. J. (1978). Serologic typing of rickettsiae of the spotted fever group by micro-immunofluorescence. J Immunol 121, 1961-1968.

Raoult, D., Fournier, P. E., Eremeeva, M. \& 7 other authors (2005). Naming of rickettsiae and rickettsial diseases. Ann N Y Acad Sci 1063, $1-12$.
Roux, V. \& Raoult, D. (2000). Phylogenetic analysis of members of the genus Rickettsia using the gene encoding the outer-membrane protein rOmpB (ompB). Int J Syst Evol Microbiol 50, 1449-1455.

Sekeyova, Z., Roux, V. \& Raoult, D. (2001). Phylogeny of Rickettsia spp. inferred by comparing sequences of 'gene D', which encodes an intracytoplasmic protein. Int J Syst Evol Microbiol 51, 1353-1360.

Takada, N., Fujita, H., Yano, Y., Ishiguro, F., Iwasaki, H. \& Masuzawa, T. (2001). First records of tick-borne pathogens, Borrelia, and spotted fever group Rickettsiae in Okinawajima Island, Japan. Microbiol Immunol 45, 163-165.

Uchida, T., Uchiyama, T., Kumano, K. \& Walker, D. H. (1992), Rickettsia japonica sp. nov., the etiological agent of spotted fever group rickettsiosis in Japan. Int J Syst Bacteriol 42, 303-305.

Yano, Y., Fujita, H. \& Takada, N. (2004). Ultrastructure of a Japanese rickettsial strain genetically identified as Rickettsia helvetica which was originally found in Europe. Microbiol Immunol 48, $535-539$. 